\title{
Quantum electrodynamics of bimolecular multiphoton processes in the condensed phase
}

\author{
Gediminas Juzeliūnas *,1, David L. Andrews \\ School of Chemical Sciences, University of East Anglia, Norwich, NR4 7TJ, UK
}

Received 2 March 1995

\begin{abstract}
Bimolecular photophysics is an area that accommodates a wide range of pairwise interactions of molecules with radiation, including cooperative absorption and emission, and bimolecular scattering. In this paper a QED theory is developed to deal with bimolecular multiphoton processes without recourse to the usual dilute medium approximation. The theory is fully microscopic, taking explicit account of effects due to any surrounding molecular medium. To this end, the concept of medium-dressed photons (bath polaritons) has been adopted, extending both to the real incoming and outgoing photons and also to the virtual photons that carry an energy mismatch between participating molecules. Modifications of the bimolecular rates resulting from the influence of the medium originate in two ways. One, associated with the real photons, brings in the refractive indexes at the appropriate photon frequencies. All terms constituting the transition matrix element are subject to introduction of the same refractive factor for each absorbed or emitted photon. Another medium effect, due to the virtual photons, appears through the electromagnetic coupling tensor which experiences, inter alia, changes due to screening and local field factors. Here the refractive effects in each term relate to the appropriate mismatch frequencies. These frequencies generally lie in a different spectral region to the real photons, and may equally be situated in either transparent or absorbing areas. In the latter absorbing case, an exponential decay factor emerges in the coupling tensor, regularising the long-range $R^{-2}$ contribution. That solves the problem of potentially infinite ensemble rates which arises in the dilute medium approximation.
\end{abstract}

\section{Introduction}

There is a wide range of phenomena resulting from the cooperative absorption, emission or scattering of photons by molecules, atoms, ions etc. For example, two species (A and B) initially in their ground electronic states can together be promoted to

\footnotetext{
Corresponding author.

1 Present address: Institute of Theoretical Physics and Astronomy, A. Goßtauto 12, Vilnius, Lithuania.
}

excited electronic states through the concerted absorption of one [1-3] or two [4-7] laser photons, as

$$
\begin{aligned}
& \mathrm{A}+\mathrm{B}+h \nu_{1} \rightarrow \mathrm{A}^{*}+\mathrm{B}^{*}, \\
& \mathrm{~A}+\mathrm{B}+h \nu_{1}+h \nu_{2} \rightarrow \mathrm{A}^{*}+\mathrm{B}^{*} .
\end{aligned}
$$

Such processes (1.1) or (1.2) are characterised by absorption at wavelengths where neither component A nor B displays intrinsic absorption, so that the cooperative absorption effect unequivocally signifies the interaction: the single-photon case is illustrated in Fig. 1. In the language of quantum electrodynamics (QED), the excess (or deficit) of energy carried 


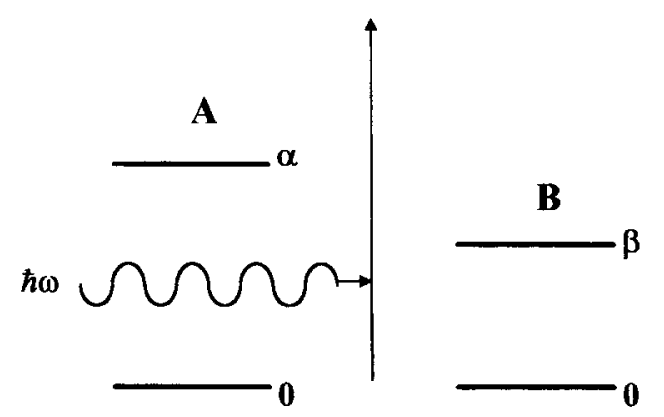

Fig. 1. Bimolecular single-photon absorption at the sum frequency.

by the incident photon annihilated at $A(B)$ is transferred to another molecule B (A) through a virtual photon [1-7].

Virtual photon coupling is a common feature of all bimolecular photophysical processes, other examples being resonance intermolecular transfer of excitation energy [8-14], bimolecular Raman scattering [15], bimolecular three-photon absorption [16], etc. Within such a classification, resonance energy transfer,

$\mathrm{A}^{*}+\mathrm{B} \rightarrow \mathrm{A}+\mathrm{B}^{*}$,

appears as the lowest-order process, as it entails no incident photons. In its QED formulation, this emerges as a second-order perturbational process mediated by intermolecular propagation of a virtual photon [8-14]. The advantage of such a quantum electrodynamical treatment is that it extends the traditional Förster theory of short-range (near-zone) dipole-dipole radiationless transfer to arbitrary A-B separations $R$, thus establishing a continuous connection with long-range (far-zone) radiative transfer.

Until recently, the QED representation of bimolecular photophysical processes has been cast in terms of a coupling between the radiation field and a pair of participating molecules $\mathrm{A}$ and $\mathrm{B}$, not taking into account the influence of other species constituting the surrounding medium. That dilute gas approximation leads to problems in the pair rates calculated for these cooperative processes, which contain terms with $R^{-6}, R^{-4}$ and $R^{-2}$ distance dependence. The latter (long-range) $R^{-2}$ contribution may result in potentially infinite total rates if directly applied to the ensemble [13]. With respect to energy transfer, that problem has recently been solved by the systematic inclusion of effects by the surrounding medium on the basis of a microscopic many-body formulation of the QED [17]. Specifically, the energy transfer was considered to be mediated by bath polaritons (medium-dressed photons), rather than vacuum photons. The reformulated theory accommodates, inter alia, the case of an absorbing medium, and a Beer's law exponential decay factor intrinsically emerges to regularise the long-range $R^{-2}$ behaviour. In addition, local field and screening effects have been properly reflected.

Following the progress on energy transfer [17], here a general microscopic theory is presented that addresses a wide variety of bimolecular multiphoton processes in the condensed phase. A distinctive feature of the present study is that not only the mediating virtual photons, but also the real incoming and outgoing photons, are here considered to be "dressed" by the medium. For $n$-photon bimolecular processes with $n \geq 1$, the medium modifications appear to be substantially more complex than those for energy transfer: nevertheless, it will be shown that the problem associated with the long-range $R^{-2}$ behaviour can here be resolved on a similar basis. A brief outline of the paper is as follows. In the next section the system Hamiltonian is presented and rate equations for the bimolecular processes are defined, followed by consideration of particular processes in Sections 3 and 4 . The concluding section summarises the results.

\section{General formulation}

\subsection{The Hamiltonian}

We shall consider a condensed molecular (or atomic) system in interaction with a quantised electromagnetic field. To deal with bimolecular multiphoton processes associated with a particular pair of molecules $\mathrm{A}-\mathrm{B}$, the full system is divided into two parts, one subsystem comprising the species $A$ and $B$, and another the "bath". The latter bath consists of the quantised radiation field and the remaining molecules which constitute the surrounding medium. The medium molecules may, but do not necessarily, 
differ in type from A and B. The system Hamiltonian then reads, in the electric dipole approximation

$H=H^{0}+V$,

$H^{0}=H_{\mathrm{A}}+H_{\mathrm{B}}+H_{\text {bath }}$,

$V=H_{\mathrm{A}}^{\text {int }}+H_{\mathrm{B}}^{\text {int }}$,

where

$H_{\text {bath }}=H_{\text {rad }}+\sum_{X \neq \mathrm{A}, \mathrm{B}}\left(H_{X}+H_{X}^{\mathrm{int}}\right)$,

with

$H_{X}^{\text {int }}=-\epsilon_{0}^{-1} \boldsymbol{\mu}(X) \cdot \boldsymbol{d}^{\perp}\left(\boldsymbol{R}_{X}\right)$.

Here $H_{\text {bath }}$ is the bath Hamiltonian, $H_{\text {rad }}$ is the unperturbed radiation Hamiltonian, $H_{X}$ is the Hamiltonian for molecule $X$ positioned at $\boldsymbol{R}_{X}$ (the molecular index $X$ spanning both the medium species, and $\mathrm{A}$ and $\mathrm{B})$ and $H_{X}^{\mathrm{int}}$ is a term representing the radiation-molecule interaction; $\boldsymbol{\mu}(X)$ is the electric dipole operator of $X$ and $\boldsymbol{d}^{\perp}\left(\boldsymbol{R}_{X}\right)$ is the microscopic operator for the transverse electric displacement field: the expansion of $\boldsymbol{d}^{\perp}\left(\boldsymbol{R}_{X}\right)$ and $H_{\text {rad }}$ in terms of radiation modes is presented elsewhere; see, e.g. Refs. $[17,20]$. Note that, in accordance with the multipolar formulation of QED [18-20] employed here, there is no instantaneous Coulomb interaction in the Hamiltonian (2.1), all intermolecular processes being mediated by the transverse quantised electromagnetic field.

Adopting a diagonal representation, the bath Hamiltonian takes the form [17]

$H_{\text {bath }}=\sum_{\sigma} \hbar \Pi_{\sigma}\left(P_{\sigma}^{+} P_{\sigma}+P_{\sigma} P_{\sigma}^{+}\right)$,

where the index $\sigma$ refers to a particular mode of bath polariton (medium-dressed photon), $P_{\sigma}^{+}$and $P_{\sigma}$ being the corresponding Bose creation and annihilation operators. At this stage we refrain from explicit analysis of these modes $\sigma$. The mode structure of the real incoming and outgoing quanta of light, which are assumed to lie in transparent regions of the linear spectrum, will be considered in the concluding part of Section 3. As regards the virtual photons, the eigenvalue analysis will be bypassed through the application of the Green's function technique developed previously [17].

\subsection{Definition of bimolecular rates}

With regards to the chosen partitioning of the system, the initial, final and all virtual intermediate states for bimolecular processes may generally be cast as

$$
\begin{aligned}
& \left.|I\rangle=\mid \text { bath }_{\mathrm{i}}\right\rangle\left|A_{0}\right\rangle\left|B_{0}\right\rangle, \\
& \left.|F\rangle=\mid \text { bath }_{\mathrm{f}}\right\rangle\left|A_{\alpha}\right\rangle\left|B_{\beta}\right\rangle, \\
& \left.\left|L_{j}\right\rangle=\mid \text { bath }_{j}\right\rangle\left|A_{r_{j}}\right\rangle\left|B_{s_{j}}\right\rangle,
\end{aligned}
$$

where $\left|A_{0}\right\rangle\left|B_{0}\right\rangle,\left|A_{\alpha}\right\rangle\left|B_{\beta}\right\rangle$ and $\left|A_{r_{j}}\right\rangle\left|B_{s_{j}}\right\rangle$ denote, respectively, the initial, final and intermediate state vectors of the selected molecular pair; $\left|b_{a t h}\right\rangle,\left|b_{i} h_{f}\right\rangle$ and $\left|b a t h h_{j}\right\rangle$ are the corresponding state vectors of the bath. In the representation of mode occupation numbers $m_{\sigma_{t}}(t=1,2,3, \ldots)$ the bath eigenvectors are given by

$$
\begin{aligned}
& \mid \text { bath }\rangle=\left|m_{\sigma_{1}}, m_{\sigma_{2}}, m_{\sigma_{3}}, \ldots\right\rangle, \\
& \mid \text { bath }\rangle=\prod_{t}\left(m_{\sigma_{t}} !\right)^{-1 / 2}\left(P_{\sigma_{t}}^{+}\right)^{m_{\sigma_{t}}}|0\rangle,
\end{aligned}
$$

10) denoting the ground (zero-polariton) state of the bath. It is to be emphasised that although Eqs. (2.7)-(2.11) resemble the standard initial conditions featuring in the QED theories on the bimolecular processes in vacuum [1-14], the state vectors $\left|m_{\sigma_{1}}, m_{\sigma_{2}}, m_{\sigma_{3}}, \ldots\right\rangle$ here no longer represent the "pure" photon states.

From application of the Fermi golden rule, the pair rate of a bimolecular process is then given by

$W_{\mathrm{FI}}=(2 \pi / \hbar)|\langle\mathrm{F}|T| \mathrm{I}\rangle|^{2} \delta\left(E_{\mathrm{F}}-E_{\mathrm{I}}\right)$,

where the transition operator $T$ may be expanded in a usual way as

$T=V+V\left(E_{1}-H^{0}+\mathrm{i} \eta\right)^{-1} V+\ldots,(\eta \rightarrow+0)$,

$E_{\mathrm{I}}$ and $E_{\mathrm{F}}$ being the energies of the initial and the final states.

Bimolecular processes can now be classified as zero-photon, one-photon, two-photon, etc. according to the number of (real) medium-dressed photons that enter or leave the system during a process '. Under such a classification, the energy transfer represented by Eq. (1.3), is obviously designated a zero-photon

\footnotetext{
'Here and henceforth, the "dressed" designation will be kept mostly implicit. The term "bath polariton" will also be used as a synonym for a dressed photon, mainly with respect to the virtual photon.
} 
process. Following the extensive analysis of the energy transfer between a pair of molecules in vacuum [8-14], the medium-induced modifications of the process have also been considered recently [17]. The formalism developed [17] will now be extended to higher-order bimolecular processes that involve real incoming and outgoing photons in addition to the virtual ones. To clarify the characteristic influences by the medium on these processes, we first consider in more detail the case of bimolecular one-photon absorption.

\section{Bimolecular one-photon absorption}

The process involves concerted excitation of both $A$ and $B$ species to their final states $\alpha$ and $\beta$ through the absorption of a single laser photon $\sigma_{1}$. The initial and final states of the radiation field are then given by

$\mid$ bath $\left._{\mathrm{i}}\right\rangle=\left|m_{\sigma_{1}}\right\rangle$, $\mid$ bath $\left._{\mathrm{f}}\right\rangle=\left|(m-1)_{\sigma_{1}}\right\rangle$.

The photon energy should be off-resonance with respect to single-photon transitions of each member of the absorbing pair so that the cooperative process occurs through intermolecular transfer of the energy mismatch by a virtual polariton (see Fig. 2). Conservation of energy requires that

$\hbar \Pi_{\sigma_{1}}=\hbar \omega_{\alpha 0}+\hbar \omega_{\beta 0}$,

where $\hbar \omega_{\alpha 0}$ and $\hbar \omega_{\beta 0}$ are the molecular excitation energies

$\hbar \omega_{\alpha 0}=E_{\mathrm{A}_{0}}-E_{\mathrm{A}_{0}}, \hbar \omega_{\beta 0}=E_{\mathrm{B}_{\beta}}-E_{\mathrm{B}_{0}}$.

As a perturbational process of third order, onephoton bimolecular absorption proceeds through two intermediate states associated with three photon events: annihilation of an incident photon, creation of a virtual polariton and annihilation of the virtual polariton. For instance, the diagram presented in Fig. 2 corresponds to the following set of intermediate states:

$\left|L_{1}\right\rangle=\left|(m-1)_{\sigma_{1}}\right\rangle\left|A_{r}\right\rangle\left|B_{0}\right\rangle$

and

$\left|L_{2}\right\rangle=\left|1_{\sigma},(m-1)_{\sigma_{1}}\right\rangle\left|A_{\alpha}\right\rangle\left|B_{0}\right\rangle$.

In this route to the final state, the incident mediumdressed photon $\sigma_{1}$ is first absorbed by molecule $\mathrm{A}$,

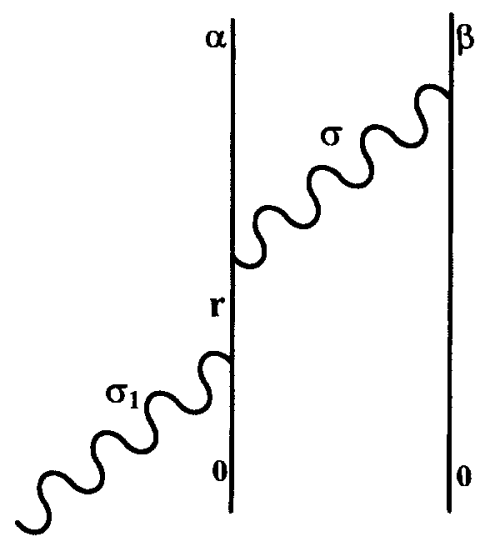

Fig. 2. Virtual photon mediation of bimolecular single-photon absorption. In this diagram the incident photon is annihilated at site $\mathrm{A}$.

promoting it to some intermediate state $\left|A_{r}\right\rangle$. Next a virtual polariton $\sigma$ is emitted by $\mathrm{A}$, accompanied by the molecular transition $\left|A_{r}\right\rangle \rightarrow\left|A_{\alpha}\right\rangle$. Finally, the virtual polariton is absorbed by molecule $\mathrm{B}$, exciting it to state $\left|B_{\beta}\right\rangle$. Note that the virtual polariton is not represented as belonging to any specific mode since the theory requires summation to be subsequently performed over all modes $\sigma$.

Altogether there are 12 different diagrams associated with one-photon bimolecular absorption [1]. By summing up all these third-order contributions one finds the following result, expressed with the convention of summation over repeated tensor indices:

$$
\begin{aligned}
\left\langle\mathrm{F}\left|T^{(3)}\right| \mathrm{I}\right\rangle= & -\varepsilon_{0}^{-1} m_{\sigma_{1}}^{1 / 2}\left[\mu_{1}^{\beta 0}(\mathrm{~B}) \theta_{l p}\left(\omega_{\beta 0}\right)\right. \\
& \times S_{j p}^{\alpha 0}(\mathrm{~A})\left\langle 0\left|d_{j}^{\perp}\left(\boldsymbol{R}_{\mathrm{A}}\right)\right| \sigma_{1}\right\rangle \\
& +\mu_{1}^{\alpha 0}(\mathrm{~A}) \theta_{p l}\left(\omega_{\alpha 0}\right) \\
& \left.\times S_{j p}^{\beta 0}(\mathrm{~B})\left\langle 0\left|d_{j}^{\perp}\left(\boldsymbol{R}_{\mathrm{B}}\right)\right| \sigma_{1}\right\rangle\right],
\end{aligned}
$$

where $\left|\sigma_{1}\right\rangle$ is an abbreviation for $\left|1_{\sigma_{1}}\right\rangle^{2}$,

$$
\begin{aligned}
\theta_{l p}(\omega) & \frac{1}{\hbar \varepsilon_{0}^{2}} \sum_{\sigma}\left(\frac{\left\langle 0\left|d_{l}^{\perp}\left(\boldsymbol{R}_{\mathrm{B}}\right)\right| \sigma\right\rangle\left\langle\sigma\left|d_{p}^{\perp}\left(\boldsymbol{R}_{\mathrm{A}}\right)\right| 0\right\rangle}{\omega-\Pi_{\sigma}+\mathrm{i} \eta}\right. \\
& \left.-\frac{\left\langle 0\left|d_{p}^{\perp}\left(\boldsymbol{R}_{\mathrm{A}}\right)\right| \sigma\right\rangle\left\langle\sigma\left|d_{l}^{\perp}\left(\boldsymbol{R}_{\mathrm{B}}\right)\right| 0\right\rangle}{\omega+\Pi_{\sigma}-\mathrm{i} \eta}\right)
\end{aligned}
$$

\footnotetext{
${ }^{2}$ In Eq. (3.6) use is made of the relation $\left\langle(m-1)_{\sigma_{1}}\right| d^{\perp}$ $\left(\boldsymbol{R}_{x}\right)\left|m_{\sigma_{1}}\right\rangle \equiv m_{\sigma_{1}}^{1 / 2}\left\langle 0\left|d^{\perp}\left(R_{x}\right)\right| \sigma_{1}\right\rangle$.
} 
is the electromagnetic dipole-dipole tensor that couples the sites $A$ and $B$, and

$S_{j p}^{\alpha 0}(\mathrm{~A})$

$$
=\frac{1}{\hbar} \sum_{r}\left(\frac{\mu_{j}^{\alpha r}(\mathrm{~A}) \mu_{p}^{r 0}(\mathrm{~A})}{\omega_{\alpha r}-\Pi_{\sigma_{1}}+\mathrm{i} \eta}-\frac{\mu_{p}^{\alpha r}(\mathrm{~A}) \mu_{j}^{r 0}(\mathrm{~A})}{\omega_{r 0}-\Pi_{\sigma_{1}}-\mathrm{i} \eta}\right)
$$

is the second-rank response tensor of the molecule A, the sum being taken over intermediate states $|r\rangle$. The corresponding second-rank molecular tensor of $\mathrm{B}, S_{j p}^{\beta 0}(\mathrm{~B})$, is defined in an analogous manner, substituting in Eq. (3.8) B for $\mathrm{A}, \beta$ for $\alpha$, and the intermediate state index $s$ for $r$. The first term in square brackets of (3.6) represents the contribution from six diagrams where the incident dressed photon is annihilated at molecule A (as in Fig. 2) and a virtual polariton transfers the energy difference of $\hbar \omega_{\beta 0}$ to molecule B. Consequently $\omega_{\beta 0}$ appears as the argument of the interaction tensor. The second term of (3.6) is due to the other six diagrams in which the incident photon is annihilated at $B$. The previous theories [1-3] neglected the effects by the medium molecules. In such a "vacuum" case the polariton modes reduce to the usual photon modes, $\sigma=(k, \lambda)$, characterised by wavevector $\boldsymbol{k}$ and polarisation $\lambda$, with the dispersion relation $\Pi_{\sigma}=c k$.

Let us now analyze the characteristic influences of the intervening medium on the rates of bimolecular photon absorption. It is apparent that two types of medium effects appear in Eq. (3.6) for $T^{(3)}$.

(i) The first type is due to the electromagnetic coupling tensor $\theta_{l p}(\omega)$. The influence of the medium on the tensor has been analyzed in detail in our previous paper on energy transfer, giving [17]

$\theta_{l p}(\omega)=\frac{1}{n^{2}}\left(\frac{n^{2}+2}{3}\right)^{2} \theta_{l p}^{\mathrm{vac}}(n \omega), n \equiv n(\omega)$,

where

$$
\begin{aligned}
\theta_{l p}^{\mathrm{vac}}(y)= & \frac{y^{3} \mathrm{e}^{i y / R / c}}{4 \pi \varepsilon_{o} c^{3}}\left[\left(\delta_{l p}-3 \hat{R}_{l} \hat{R}_{p}\right)\right. \\
& \left.\times\left(\frac{c^{3}}{y^{3} R^{3}}-\frac{\mathrm{i} c^{2}}{y^{2} R^{2}}\right)-\left(\delta_{l p}-\hat{R}_{l} \hat{R}_{p}\right) \frac{c}{y R}\right]
\end{aligned}
$$

(with $\hat{\boldsymbol{R}}=\boldsymbol{R} / R$ and $\boldsymbol{R}=\boldsymbol{R}_{\mathrm{A}}-\boldsymbol{R}_{\mathrm{B}}$ ) has the structure of the familiar tensor for the retarded dipole-dipole coupling in vacuum, its argument $y=n \omega$ being now scaled by the (frequency dependent) refractive index $n \equiv n(\omega)=n^{\prime}+\mathrm{i} n^{\prime \prime}=\varepsilon_{r}^{1 / 2}$. Here $\varepsilon_{r}$ is the complex relative dielectric permittivity satisfying the Clausius-Mossotti relation [17]. The full details including the derivation of Eq. (3.9) through a microscopic Green's function analysis has been given elsewhere [17]. This development has made it possible to accommodate an arbitrary number of energy levels for each molecule of the medium, in contrast to the two-level representation employed by Knoester and Mukamel in a related study on intermolecular forces and superradiance [21]. Accordingly the theory applies to media with various types of energy spectrum, including those possessing smooth and dense absorption bands. In other words, the above tensor $\theta_{l p}(\omega)$ is equally applicable for description of both transparent and absorbing areas of the spectrum. In the latter absorbing case $\left(n^{\prime \prime} \neq 0\right)$ an exponential decay factor $\exp \left(-n^{\prime \prime} \omega R / c\right)$ features in the tensor (3.9), in addition to the oscillatory factor $\exp \left(i n^{\prime} \omega R / c\right)$. This solves the problem of potential divergences in the ensemble rates both for energy transfer and also for the multiphoton processes under present consideration.

The tensor (3.9) exhibits, besides the exponential factor $\exp (\mathrm{i} n \omega R / c)$, the local field and screening contributions: the latter contributions vary from $n^{-2}\left[\left(n^{2}+2\right) / 3\right]$ in the near-zone to $\left[\left(n^{2}+2\right) / 3\right]$ in the far-zone. It is to be noted that the $\omega$-dependent refractive index entailed in the $\theta_{l p}$ tensor is to be calculated for the two frequencies $\omega_{\beta 0}$ and $\omega_{\alpha 0}$ in each term of Eq. (3.6), corresponding in each case to the energy mismatch transferred across from the site of incident photon annihilation. This is due to the two-centre character of the bimolecular absorption process.

(ii) The second type of medium influence emerges through the matrix elements $\left\langle 0\left|d_{j}^{\perp}\left(\boldsymbol{R}_{X}\right)\right| \sigma_{1}\right\rangle(X=$ $\mathrm{A}, \mathrm{B})$ that enter the transition matrix element (3.6). These are the contributions associated with the real "dressed" photons $\sigma_{1}$ that are assumed to fall into the transparent region of the linear spectrum, in which $n^{\prime \prime} \neq 0$. The "dressed" photon $\sigma_{1}$ carries the frequency

$\Pi_{\sigma_{1}}=c k_{1} / n\left(\Pi_{\sigma_{1}}\right)$ 
and is characterised by its quasi-momentum $\boldsymbol{k}_{1}$, two polarisation components $\lambda_{1}=1,2$, as well as an extra index $m$ that labels branches of polariton dispersion [22]. The matrix element $\left\langle 0\left|d_{j}{ }^{\perp}\left(\boldsymbol{R}_{X}\right)\right| \sigma_{1}\right\rangle$ can be shown [22] to take the following form:

$$
\begin{aligned}
\left\langle 0\left|d_{j}^{\perp}\left(\boldsymbol{R}_{X}\right)\right| \sigma_{1}\right\rangle & \\
= & \frac{\mathrm{i}}{n^{1 / 2} n_{\mathrm{g}}^{1 / 2}}\left(\frac{n^{2}+2}{3}\right)\left(\frac{\hbar \Pi_{\sigma_{1}} \varepsilon_{0}}{2 V_{0}}\right)^{1 / 2} \\
& \times e_{j}^{\left(\lambda_{1}\right)}\left(k_{1}\right) \mathrm{e}^{\mathrm{i} k_{1} \cdot \boldsymbol{R}_{X}},
\end{aligned}
$$

where $V_{0}$ is the quantisation volume and $e^{\left(\lambda_{1}\right)}\left(k_{1}\right)$ is a unit vector for one of the two transverse polarisations $\left(\lambda_{1}=1,2\right)$ of the photon. Here also $n \equiv n\left(\Pi_{\sigma_{1}}\right)$ is the (phase) refractive index. Another group refractive index $n_{\mathrm{g}} \equiv n_{\mathrm{g}}\left(\Pi_{\sigma_{1}}\right)$, characterising the group velocity of light as $v_{\mathrm{g}}=c / n_{\mathrm{g}}$, is given by

$n_{\mathrm{g}}\left(\Pi_{\sigma_{1}}\right)=\frac{\mathrm{d}\left[\Pi_{\sigma_{1}} n\left(\Pi_{\sigma_{1}}\right)\right]}{\mathrm{d} \Pi_{\sigma_{1}}}$.

In this way, the matrix element $\left\langle 0\left|d_{j}^{\perp}\left(\boldsymbol{R}_{X}\right)\right| \sigma_{1}\right\rangle$ experiences refractive modifications that are independent of the particular site $X=\mathrm{A}, \mathrm{B}$. In other words, both terms of Eq. (3.6) are subject to introduction of the same refractive factor $n_{\mathrm{g}}^{-1 / 2} n^{-1 / 2}\left[\left(n^{2}\right.\right.$ $+2) / 3$, where both $n$ and $n_{\mathrm{g}}$ are evaluated at frequency $\Pi_{\sigma_{1}}$ of the external photon $\sigma_{1}$. Noting the linear dependence of $\left|T^{(3)}\right|^{2}$ on the group velocity $c / n_{\mathrm{g}}$, one arrives at the $n^{-1}\left[\left(n^{2}+2\right) / 3\right]^{2}$ refractive dependence of the bimolecular rate per unit photon flux, which is consistent with the result for ordinary linear (monomolecular) absorption [22,23].

\section{Extension to other bimolecular processes}

Before considering higher-order bimolecular processes, we first note a straightforward extension of the above one-photon theory to bimolecular (thirdorder) spontaneous emission, the process in which two excited molecules undergo cooperative downward transitions to their ground state through emission of a photon. Such a process has a potential role as the relaxation channel in photonic band-gap crystals [24], where direct first-order spontaneous emission is suppressed. The third-order spontaneous emission may be regarded as a time reversal of the bimolecular cooperative absorption depicted in Fig. 2. Accordingly, the associated transition matrix element follows from Eq. (3.6) by reversing the momentum of the real photon, also setting $m_{\sigma_{1}}=1$. Here one arrives at an $n\left[\left(n^{2}+2\right) / 3\right]^{2}$ refractive dependence for the rate of third-order spontaneous emission. That is in agreement with the result for ordinary (monomolecular) spontaneous emission

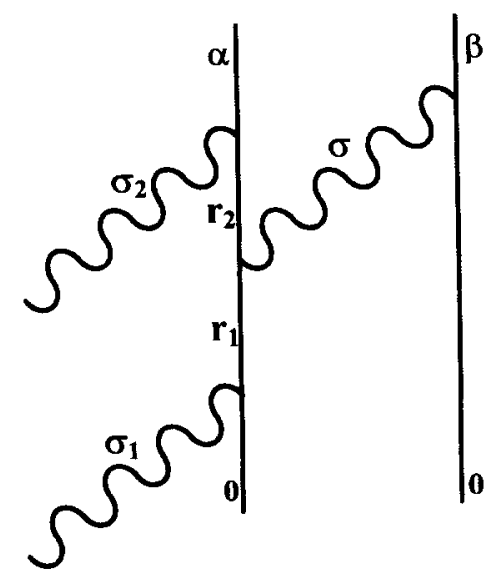

(a)

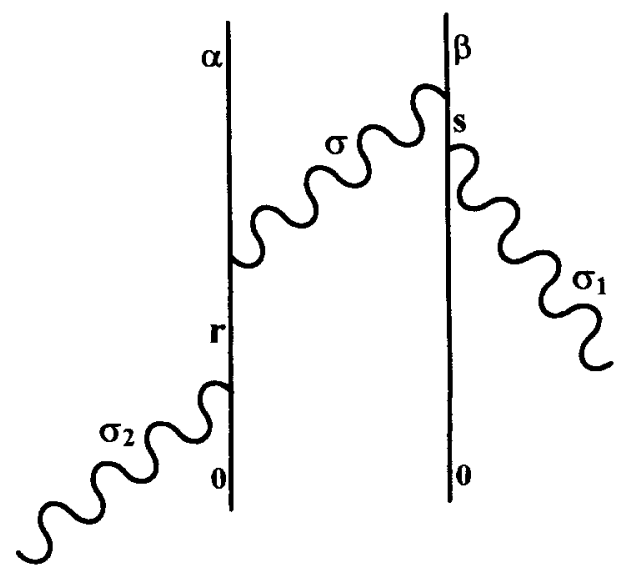

(b)

Fig. 3. Typical time-ordered diagrams for bimolecular two-photon absorption, (a) where the two real incident photons are annihilated at one site; (b) where one incident photon is annihilated at each site. 
[21-23]. Distinctive features of the third-order process are reflected in the additional refractive modifications due to coupling tensors $\theta_{l p}\left(\omega_{\alpha 0}\right)$ and $\theta_{l p}\left(\omega_{\beta O}\right)$ in Eq. (3.6).

Consider next the concerted excitation of a molecular pair through the absorption of two laser photons. Bimolecular two-photon absorption emerges from the fourth order of the perturbation expansion. The complete set of interaction sequences is accounted for by 96 different time-ordered diagrams [4], typical examples being shown in Fig. 3. For the vacuum case, the resulting fourth-order matrix element of the transition operator is presented in Ref. [4] by Eq. (5.13) ${ }^{3}$. As in the case of one-photon absorption, the transition matrix element for bimolecular absorption is a linear combination of dipole-dipole interaction tensors $\theta_{l p}\left(\omega_{X}\right),\left(\omega_{X}=\right.$ $\left.\omega_{\beta 0}, \ldots\right)$ weighted by a radiation tensor for the incident photon polarisation field, and also the molecular tensors ${ }^{4}$. The same structure emerges in the transition matrix elements for bimolecular Raman scattering [15], three-photon bimolecular absorption, and other two-centre processes such as the various manifestations of chiral discrimination associated with coupled chromophores $[20,25]$.

It is therefore fairly straightforward to extend the Section 3 analysis of medium influences on bimolecular single-photon absorption to these higher-order bimolecular processes. The familiar transition matrix elements of the previously considered vacuum response are modified in real media as follows. The vacuum electromagnetic tensors featured in the conventional formulae are to be replaced by their medium counterparts through Eq. (3.9), where in each case the refractive index is to be calculated at the frequency corresponding to the appropriate mismatch energy. For terms representing mechanisms for which the energy mismatch falls into an absorbing region of the spectrum, the imaginary part of the refractive index automatically ensures the appearance of an

\footnotetext{
' In Ref. [4] there should be no complex conjugation bars over $V_{k l} \equiv \theta_{k !}^{\text {vac }}$ in the fourth line of Eq. (5.13), and the modulus should be taken of $\omega_{\beta 0}-\omega_{i}(i=1,2)$ in the arguments of $V_{k l}$ in the first two lines.

4 The molecular tensors now include the third-rank $\chi$, whose structure has been detailed in Ref. [4], in addition to the secondrank $S$ already featured in the single-photon case.
}

exponential decay factor of the form $\exp \left(-n^{\prime \prime} \omega R / c\right)$, associated with energy losses: this type of factor occurs in addition to the oscillatory (phase) factor $\exp \left(\mathrm{i} n^{\prime} \omega R / c\right)$ which arises in any (absorbing or loss-less) medium. The presence of this factor results in different distance dependent phases for each term contributing to the transition matrix element. Finally, each real photon brings in the same refractive factor $n^{-1 / 2} n_{\mathrm{g}}^{-1 / 2}\left[\left(n^{2}+2\right) / 3\right]$, to be calculated at the photon frequency, into all terms entering the transition matrix element.

\section{Summary}

A QED theory has been developed to deal with bimolecular multiphoton processes without recourse to the dilute gas approximation. The theory is fully microscopic, taking explicit account of effects due to the surrounding molecular medium. To this end, the concept of medium-dressed photons (bath polaritons) has been adopted, extending both to the real incoming and outgoing photons and also to the virtual photons that carry the energy mismatch between the participating molecules. Modifications of the bimolecular rates resulting from the influence of the medium originate in two ways. One, associated with the real photons, brings in the refractive indexes at the appropriate photon frequencies. Another refractive effect, due to the virtual photons, appears through the electromagnetic coupling tensor which experiences changes due to screening and local field factors, and also $n$-scaling of its argument as featured in Eq. (3.9). Accordingly in absorbing media, an exponential decay factor $\exp \left(-n^{\prime \prime} \omega R / c\right)$ emerges, thus regularising the long-range $R^{-2}$ contribution.

\section{Acknowledgements}

This work was supported by a grant from the Engineering and Physical Sciences Research Council.

\section{References}

[1] E. Hudis, Y. Ben-Aryeh and U.P. Oppenheim, Phys. Rev. A 43 (1991) 3631. 
[2] G. Kweon and N.M. Lawandy, Phys. Rev. B 49 (1994) 4445.

[3] D.L. Andrews and P. Allcock, Chem. Phys. Letters 231 (1994) 206.

[4] D.L. Andrews and K.P. Hopkins, Advan. Chem. Phys. 77 (1990) 39

[5] D.L. Andrews and N.P. Blake, J. Mod. Opt. 37 (1990) 701.

[6] D.L. Andrews and A.M. Bittner, Chem. Phys. 165 (1992) 1.

[7] D.L. Andrews and A.M. Bittner, Anal. Proc. 29 (1992) 333.

[8] E.A. Power and T. Thirunamachandran, Phys. Rev. A 28 (1983) 2671.

[9] D.L. Andrews and B.S. Sherborne, J. Chem. Phys. 86 (1987) 4011.

[10] D.L. Andrews, D.P. Craig and T. Thirunamachandran, Intern. Rev. Phys. Chem. 8 (1989) 37.

[11] D.L. Andrews, Chem. Phys. 135 (1989) 195.

[12] D.L. Andrews and G. Juzeliūnas, J. Chem. Phys. 95 (1991) 5513.

[13] D.L. Andrews and G. Juzeliūnas, J. Chem. Phys. 96 (1992) 6606 .
[14] D.P. Craig and T. Thirunamachandran, Chem. Phys. 167 (1992) 229.

[15] D.L. Andrews and N.P. Blake, Phys. Rev. A 41 (1990) 2547.

[16] D.L. Andrews and A.M. Bittner, J. Phys. B 26 (1993) 675.

[17] G. Juzeliūnas and D.L. Andrews, Phys. Rev. B 49 (1994) 8751.

[18] E.A. Power and S. Zienau, Phil. Trans. Roy. Soc. London A 251 (1959) 427.

[19] R.G. Woolley, Proc. Phys. Soc. London A 321 (1971) 557.

[20] D.P. Craig and T. Thinunamachadran, Molecular quantum electrodynamics (Academic Press, New York, 1984).

[21] J. Knoester and S. Mukamel, Phys. Rev. A 40 (1989) 7065.

[22] G. Juzeliūnas, Chem. Phys. 198 (1995) 145.

[23] V.M. Agranovich and M.D. Galanin, Electronic excitation energy transfer in condensed matter (North-Holland, Amsterdam, 1982).

[24] S. John and J. Wang, Phys. Rev. B 43 (1991) 12772.

[25] D.L. Andrews, Faraday Discussions, 99 (1995) 375. 